\title{
Clinical utility of home videos for diagnosing epileptic seizures: a systematic review and practical recommendations for optimal and safe recording
}

\author{
Lorenzo Ricci $^{1}$ - Marilisa Boscarino ${ }^{1}$. Giovanni Assenza ${ }^{1} \cdot$ Mario Tombini $^{1} \cdot$ Jacopo Lanzone ${ }^{1} \cdot$ Vincenzo Di Lazzaro $^{1}$. \\ Sara Casciato ${ }^{2}$ - Alfredo D'Aniello ${ }^{2}$. Alessandra Morano ${ }^{3}$ - Giancarlo Di Gennaro ${ }^{2}$ (D) - Epilepsy Study Group of the \\ Italian Neurological Society
}

Received: 8 September 2020 / Accepted: 4 January 2021 / Published online: 20 January 2021

(C) Fondazione Società Italiana di Neurologia 2021

\begin{abstract}
Background The aim of the present systematic revision is to analyze existing published reports about the use of home-videos recordings (HVRs) to support physicians in the differential diagnosis of paroxysmal seizure-like episodes (PSLE). We also developed practical recommendations in order to ensure adequate quality standards and safety advice for HVRs.

Material and methods A comprehensive search of PubMed, Medline, Scopus, and Google Scholar was performed, and results were included up to July 2020. All studies concerning the use of HVRs as a diagnostic tool for patients presenting PSLE were included.

Results Seventeen studies satisfied all inclusion and exclusion criteria and were considered for the review. A consistent boost in diagnostic and clinical decision-making was reported across all studies in the literature. One study found that HVRs decreased the stress level in many families and improved their quality of life. Training in performing good-quality videos is necessary and increases the diagnostic value of HVRs.

Conclusions HVRs can be of diagnostic value in epilepsy diagnosis and management. HVRs are low cost, widespread, and may provide great support for neurologists. It is important to train patients and caregivers in performing good quality videos to optimize this useful tool and to guarantee safety standards during the recording.
\end{abstract}

Keywords Home videos $\cdot$ Seizures $\cdot$ Differential diagnosis $\cdot$ Smartphone $\cdot$ Paroxysmal nonepileptic events

\section{Background}

Epilepsy is a clinical diagnosis, and the accurate characterization of seizures is of utmost importance for prognosis and for the appropriate choice of treatment $[1,2]$. Differential diagnosis for seizures is broad. Even experienced neurologists can be misled when the diagnosis needs to be inferred from descriptions provided by family

Giancarlo Di Gennaro

gdigennaro@neuromed.it

1 Institute of Neurology, Neurophysiology and Neurobiology, Department of Medicine, Università Campus Bio-Medico di Roma, Rome, Italy

2 IRCSS NEUROMED, Pozzilli, IS, Italy

3 Department of Human Neurosciences, "Sapienza" University of Rome, Rome, Italy members or caregivers who witnessed the seizures. Indeed, misdiagnosis in epilepsy is not uncommon with rates varying from 4.6 to $30 \%$ across several studies [3]. Currently, neurologists are supported in the diagnosis of epilepsy by neuroimaging studies and neurophysiological techniques which allow a prolonged recording of electroencephalographic (EEG) activity. However, differential diagnosis, particularly with psychogenic nonepileptic events (PNEE) and nonepileptic paroxysmal events (NEPE), is a clinical challenge and can contribute to the rate of misdiagnoses in epilepsy [4]. In this scenario, inpatient long-term video-electroencephalographic (VEEG) monitoring has largely demonstrated its clinical value in differential diagnosis of paroxysmal events with a detection rate of epileptic seizures of almost 70\% [5]. False negatives at VEEG can be attributed to low frequency of events or to their association with specific settings that are difficult to reproduce in a hospital environment. Moreover, some patients 
with epilepsy, especially those with psychiatric comorbidities, may present both seizures and PNEE, thus complicating the diagnostic process [6]. Social and economic conditions may further represent an obstacle for access to outpatient examinations in developing countries, with a consequent delay in diagnosis [7]. Although VEEG remains the gold standard for diagnosis, a detailed clinical history and witnessed description of behavior during ictal events remains a mainstay in epilepsy diagnosis. However, witnessed description of seizures usually has low reliability as it depends mainly on the observers' level of medical knowledge [8].

In the present era of smartphone devices which are ubiquitous in society [9], there is a crescent interest in the potential role of home video recordings (HVRs) in supporting the diagnosis of epilepsy. Several studies have already highlighted their non-inferiority to VEEG monitoring to diagnose epilepsy [10-13], and many examples already exist of smartphone applications that aid physicians in clinical decision-making and optimal management of patients with epilepsy $[14,15]$. However, there is no consensus on quality standards and safety recommendations for HVRs.

The aim of the present systematic revision is to analyze existing evidence of literature where HVRs have been used to support physicians in diagnosing paroxysmal seizure-like episodes (PSLE). We also propose practical recommendations in order to ensure adequate quality standards for seizures' HVRs, which will allow the highest benefit in terms of clinical practice and diagnostic support, while providing practical safety measures to guarantee patients' security and protection.

\section{Methods}

\section{Literature search strategy and study selection process}

A systematic review was performed applying the PRISMA (Preferred Reporting Items for Systematic Reviews and Meta-Analyses) guidelines [16]. Full-text articles and conference proceedings were selected from a comprehensive search of PubMed, Medline, Scopus, and Google Scholar databases. Keywords and their synonyms were combined in each database as follows: ("home-videos" OR "smartphones") AND ("epilepsy" OR "seizure") AND ("home seizure videos" OR "domestic epilepsy videos"). No filter was applied on the publication date of the articles, and all results of each database were included up to July 2020.

After removal of duplicates, all articles were evaluated, independently, through a screening of titles and abstracts by three independent reviewers (L.R., M.T., G.A.). The same three reviewers performed an accurate reading of all full-text articles assessed for eligibility to this study, and they performed a collection of data to minimize the risk of bias. In case of disagreement among investigators regarding the selection of specific articles, the senior investigator made the final decision (G.A.).

Full-text articles were selected for systematic revision if they met the following inclusion criteria:

(i) the study included patient/s who performed HVRs because of PSLE; (ii) HVRs were utilized as a diagnostic tool; (iii) HVRs were performed using either smartphones or other types of domestic home-video recordings (i.e., cameras); (iv) articles were written in English language; (v) prospective interventional studies with or without control (active or passive) and with or without randomization; (vi) prospective and retrospective observational studies; (vii) case reports/series; (viii) monocentric and multicentric studies; (ix) articles were published in a peer-reviewed journal.

The exclusion criteria were (i) use of home videos together with EEG recordings for diagnosing PSLE; (ii) use of HVRs for indications different from diagnosing PSLE; (iii) studies conducted in animals or in vitro models; (iv) reviews, books, and conference proceedings.

\section{Data extraction process}

Database searching identified 78 articles (Fig. 1). Thirteen articles were excluded because of duplicate. Articles were exported on a separate database, and their data were independently reviewed by two experienced epileptologists (M.T. and G.A.), and by a third senior author (V.D.L) in case of disagreement. After an accurate revision of full manuscripts, 17 articles satisfied all the inclusion and exclusion criteria and were considered for evaluation. The selected articles were furtherly classified according to the following checklist: (i) authors and years; (ii) number of patients and type of publication (i.e., case reports or clinical studies); (iii) age of patients (pediatric or adult age); (iv) medical center where the study was performed; and (v) characteristics and key findings of the study.

\section{Quality and safety recommendations}

Nine epileptologists with recognized expertise in diagnostic evaluation, EEG, and management and treatment of epilepsy were gathered to address the writing of quality and safety recommendations for optimal HVRs. The expert panel discussed relevant data from the systematic review of the English-language medical literature in a series of conference calls. Critical evaluations included study design, numbers of patients, definitions used, outcomes reported, and potential biases. The committee members synthesized the data, and inconsistencies were resolved by means of discussion until a consensus was achieved. The final quality and safety recommendations were reviewed and approved by all nine participants. 
Fig. 1 Database searches

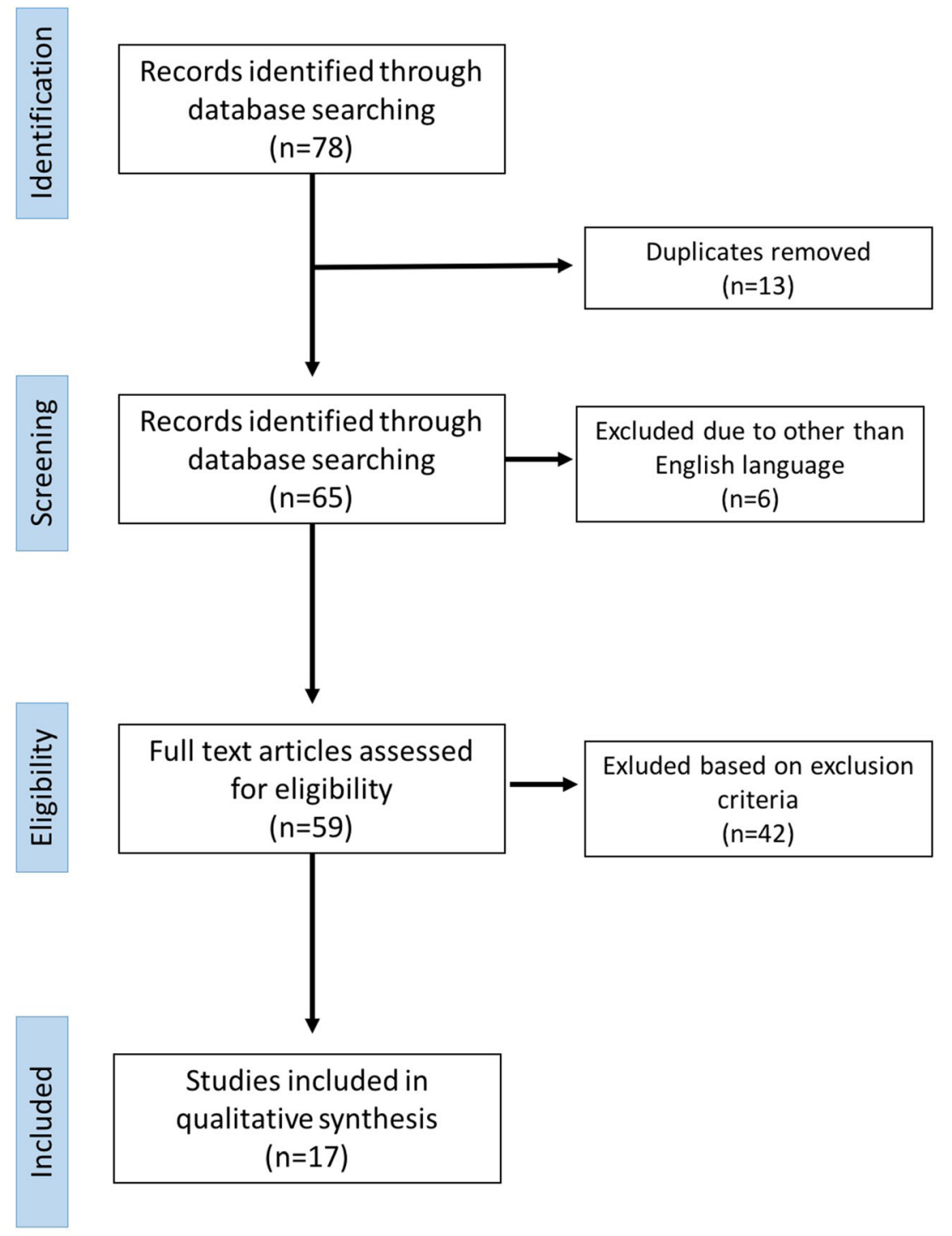

\section{Results}

Table 1 displays the 17 identified studies [10-13, 17-29] and case reports that have included clinical application of home/ smartphone videos in diagnosing PSLE. Studies were carried out in North America, Europe, and Asia. Below, we summarize relevant clinical aspects of reported studies.

The only prospective, multicenter masked clinical trial was conducted recently in 44 patients (age: $20-82$ ) by Tatum et al. [10]. They evaluated the diagnostic performances of smartphone recorded paroxysmal neurological events in 8 tertiary care epilepsy centers and compared measures of performance between board-certified expert epileptologists and neurology trainees. The diagnostic accuracy of smartphone videos interpretation for epileptic seizures was $89.1 \%$ for experts and $75.1 \%$ for residents, providing class II evidence of high diagnostic accuracy for smartphone videography.
Four articles included case reports and/or case series of HVRs supporting the diagnosis of children's spells [17], myoclonic status epilepticus [20], anoxic epileptic seizures [21], and temporal lobe epilepsy [24].

The only study investigating the potential impact on quality of life of HVRs was conducted by Johansen et al. [19]. They employed a structured questionnaire which was sent to the parents of 173 children with epilepsy and who had been recommended to a home video observation system of seizures. They concluded that the installation of a video TV observation system in a bedroom at home decreased the stress level in many families and improved their quality of life.

One study [29] was conducted only in infants using an instructional leaflet on HVRs for the general movement assessment completed by parents. The authors demonstrated the effectiveness of an instructional leaflet in guiding parental home recording of infants and the feasibility of HVRs for 


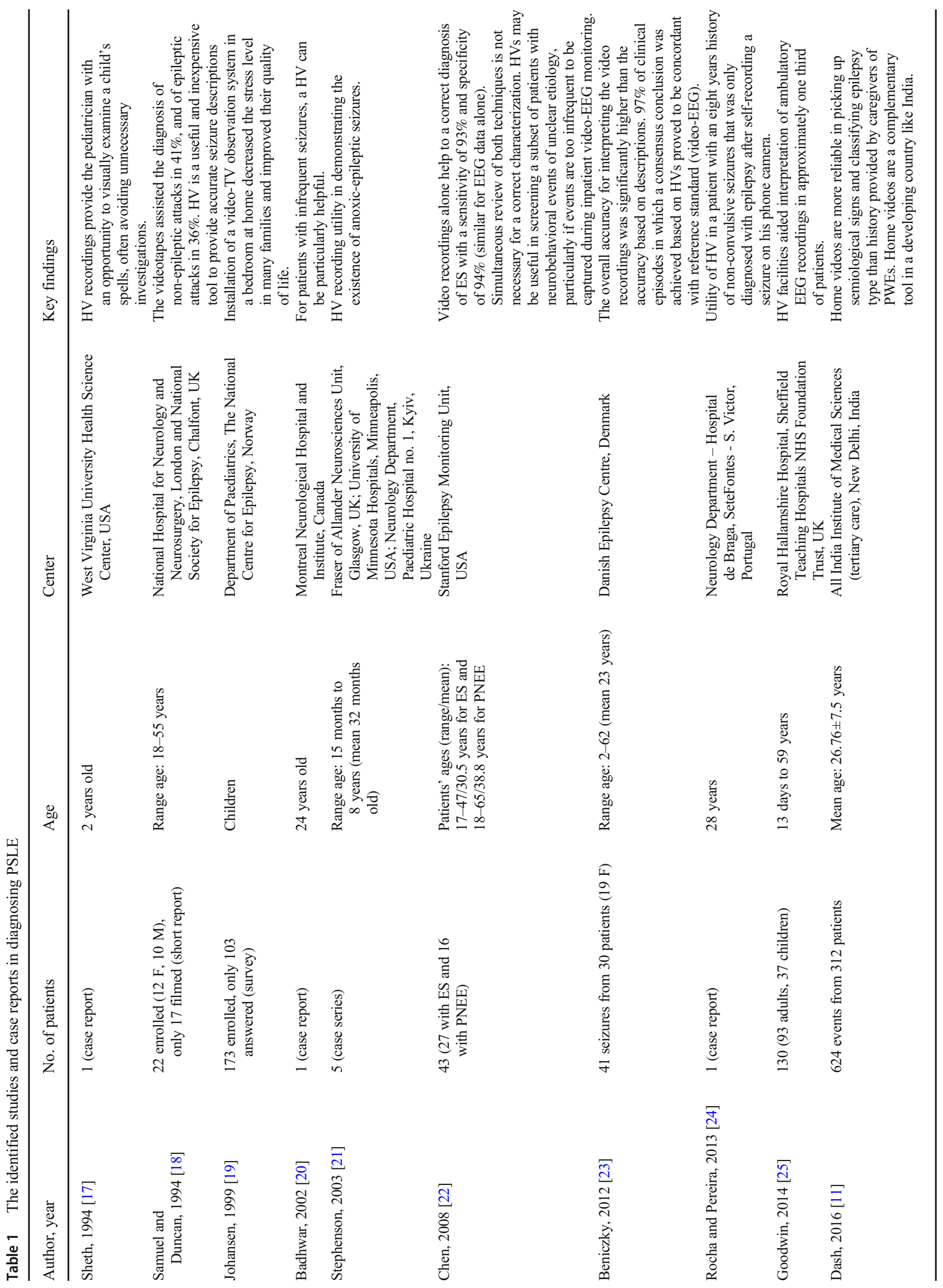




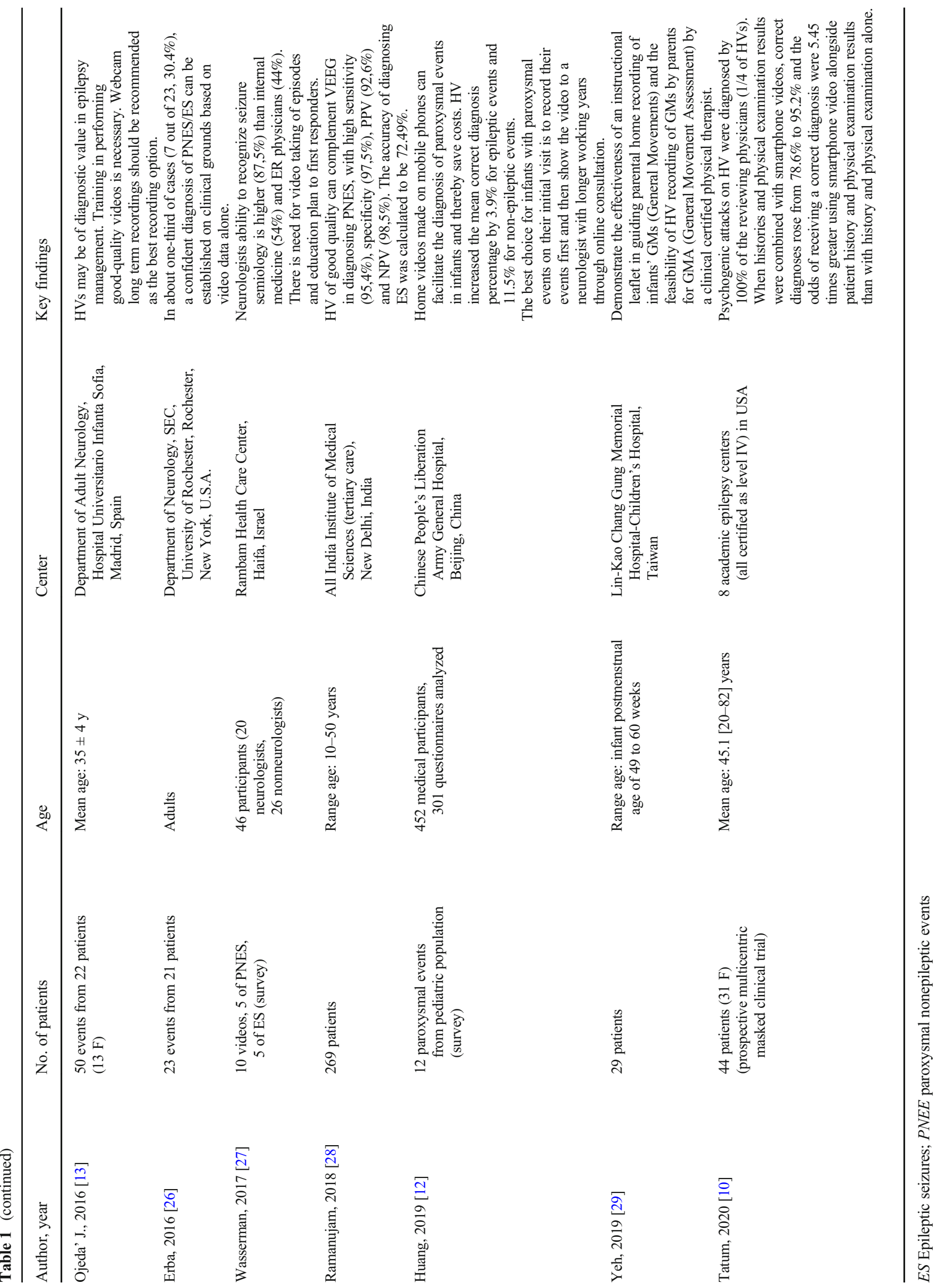


general movement assessment by a clinical certified physical therapist.

Two studies $[12,27]$ employed a video-based survey to investigate the diagnostic accuracy of HVRs among medical professionals. Huang et al. [12] analyzed 301 questionnaires collected during pediatric academic conferences using HVRs from 12 children with PSLE. HVRs increased the mean correct diagnosis percentage by $3.9 \%$ for epileptic events and $11.5 \%$ for non-epileptic events. Similarly, Wasserman and colleagues [27] showed 10 video episodes' recordings of PSLE to 46 medical participants (20 neurologists and 26 non-neurologists). Neurologists' ability to diagnose correctly seizures' semiology was higher $(87.5 \%)$ than internal medicine $(54 \%)$ and emergency department physicians (44\%), underlining the need for video taking of episodes and education plan to first responders.

Six articles [18, 22, 23, 25, 26, 28] explored the clinical value of HVRs in differentiating PNEE from epileptic seizures. Samuel and Duncan [18] reported that HVRs supported the diagnosis of PNEE and epileptic seizures in $41 \%$ and in $36 \%$ of their patients, respectively. Chen et al. [22] reported a sensitivity of $93 \%$ and a specificity of $94 \%$ for HVRs in differentiating epileptic seizures form PNEE, which was superior to EEG data alone (sensitivity $89 \%$ and specificity $94 \%$ ). Beniczky et al. [23] reported an overall accuracy of $85 \%$ in interpreting seizure semiology from HVRs. Goodwin et al. [25] found that $82 \%$ of HVRs aided the diagnosis of ambulatory EEGs performed in their center. Erba et al. [26] reported that HVRs provided an accurate diagnosis of epileptic seizures/PNEE in about one third of cases (7 out of 23, $30.4 \%$ ). Ramanujam et al. [28] found that HVRs diagnosed PNEE with a sensitivity of $95.4 \%$, a specificity of $97.5 \%$, a PPV of $92.65 \%$, and a NPV of $98.5 \%$.

One study [11] evaluated the impact of HVRs in picking up semiological signs and classifying epilepsy type in a developing country (India). The authors concluded that the widespread availability of mobile phones, even in the rural areas of India, can be harnessed to capture seizures and classify epilepsy accurately, offering a significant aid in the diagnostic classification of PSLE.

Finally, the study conducted by Ojeda and colleagues [13] was the only one to provide formal instructions to optimize the recording of PSLE in an adult epilepsy clinic. They found that training in performing good quality HVRs offered high diagnostic value in the epilepsy management of adults with epilepsy.

\section{Discussion}

This review suggests that HVRs are a promising and reliable tool for the diagnostic assessment of patients with PSLE. A consistent boost in diagnostic and clinical decision-making was reported across all studies in the literature. This suggests that HVRs are effective, timesaving, and provide consistency of care in different centers and across continents, with high practical value and universal relevance. The results of our systematic review also highlight the recommendations of the operational classification of seizure type by the International League Against Epilepsy (ILAE) [30] which encourage the use of supporting information including videos recorded by family members as part of the diagnostic process for seizures. At the same time, the reviewed articles indicate the clinical observation of PSLE is usually sufficient in differentiating seizure's semiology and differentiating epileptic seizures from PNEE, even before viewing the EEG. However, it should be mentioned that HVRs had the highest clinical value only for epileptologists, while general neurologists and nonneurologists usually required additional diagnostic tools and showed lower interrater agreement [27]. Therefore, since it has been proven that discriminating seizure's semiology is a learned skill and requires specific neurologic training [8, 31], specific education for the task may be needed in order to fully exploit the clinical benefit provided by HVRs.

\section{Clinical and care implications and future research priorities}

The most consistent added diagnostic value for HVRs was in differentiating patients with PNEE from epileptic seizures, especially when motor signs were present [18, 22, 23, 25, 26, 28]. PNEE still represent a clinical challenge even for the experienced neurologist since their manifestations are greatly variable and often resemble those of epileptic seizures [32]. The ILAE Nonepileptic Seizures Task Force published recommendations indicate that HVRs can provide some advantages, but their use is not recommended because the diagnostic yield of typical events recorded by witnesses has not been systematically evaluated [33]. However, the results of recent studies have started to challenge this notion, and we suggest that HVRs should not only be recommended but that they may also represent a "red flag" for seizure mimic if volunteered by patients, as suggested by Tatum et al. [10], probably because they respond to the need of patients to confirm that their episodes are "real". Indeed, the possibility to diagnose PNEE after the first attack at the emergency department would largely benefit this patient population as it may avoid unnecessary examinations, expedite treatment planning, and reduce the risk of undesired side effects from unnecessary antiseizure medications.

There is a lack of longitudinal data on the diagnostic trajectory of patients with PSLE recommended for home video registration of their attacks since all the reviewed studies were restricted to a single video at one point. Assessing longitudinal care with serial HVRs could reveal even greater advantages, for instance as a method of screening for patients before admission to a full long-term video EEG monitoring unit. This will eventually help reducing waiting lists and to expedite the 
selection for presurgical work-up in those cases who may benefit the most from resective epilepsy surgery [34].

Socioeconomic and demographic factors have been considered in one study only [11]. The applicability of HVRs in different healthcare systems and countries represents an opportunity for exploring patient populations where manifestations and frequency of PNEE may vary in relation to cultural differences. Furthermore, HVRs are simple, widely available, and inexpensive compared to video EEG monitoring which is available at very few centers in developing countries.

Several studies have focused on the accuracy of investigations. However, privacy regulations and ethical issues need to be addressed before developing educational websites or telemedicine services for this purpose.

Finally, further studies with larger cohorts are needed to assess the added value of HVRs for different age groups, including neonates and elderly, since the differential diagnosis across age groups may vary greatly and require customized clinical management $[35,36]$.

\section{Recommendations for quality standards of video recording and safety assessment}

There is clear need for population-based studies with welldescribed instructions for optimal video recording of PSLE. Indeed, Ojeda et al. [13] showed that the quality of home videos is of utmost importance, and caregivers of these patients should be instructed to ensure good illumination, visibility of the face as well as the rest of the body without obstruction, and capture of the entire event from the beginning. Furthermore, there is a lack of data concerning safety recommendations for HVRs, especially when motor signs are present, and there is a concrete risk of seizure's related injuries.

Hopefully, the development of training modules and detailed instructions in local languages may eventually increase the number of patients referred for home video registration of PSLE, strengthen the evidence of clinical usefulness, and guarantee adequate measures of safety standards.

Herein, we propose our practical recommendations for HVRs (Supplementary Table 1 [English version] and 2 [Italian version]). We focused on the quality standards which have been already suggested by Ojeda et al. [13] and Tatum et al. [10], including quality standards rating score and instructions in demonstrating interactivity with the patient. A key issue is the recording the episode from the onset, which may not always be possible but of utmost importance for differential diagnosis, especially when nonmotor manifestations are prevalent and nonepileptic syndromes other than PNEE are suspected since prodromal symptoms and the results of other physiological parameters (i.e., ECG telemetry, blood pressure) are often necessary to formulate a correct diagnosis [26].

As for safety instructions, we recommend checking the surrounding area for any condition favoring seizure's related injuries (i.e., head traumas or drowning) [37]. Special emphasis has been given to autonomic alterations, which can be particularly worrisome in children because of the risk of apnea and respiratory failure [38]. Last, we suggest recording each episode for no more than 2-3 min, in order to prevent the insurgence of status epilepticus [39], and follow the instruction for rescue therapy provided by the neurologist or alert the healthcare emergency service.

\section{Conclusion}

The results of this systematic review suggest that HVRs of seizures can be of diagnostic value in epilepsy diagnosis and management. HVRs are low cost, widespread, and may provide great support for neurologists. It is important to train patients and caregivers in performing good quality videos to optimize this useful tool. Home video recordings are not intended to replace video EEG monitoring studies but probably could help in selecting cases to confirm its necessity and appropriateness and in reducing waiting lists of long-term EEG monitoring. Data protection and privacy issues should be highly guaranteed to the patient by the physician and the institution.

In addition, HVRs can be especially helpful in situations when measures of lockdown, social distancing, and reduction of outpatient visits are required, as we have experienced with the recent coronavirus disease 2019 pandemic [40]. Finally, it is important to emphasize to parents and caregivers that the patient's safety comes always first, and of course, it should be constantly assured before and during the video recording.

Supplementary Information The online version contains supplementary material available at https://doi.org/10.1007/s10072-021-05040-5.

Acknowledgments We are grateful to: Umberto Aguglia, Ettore Beghi, Vincenzo Belcastro, Paolo Benna, Amedeo Bianchi, Francesca Bisulli, Vittoria Cianci, Fabrizio A. de Falco, Giovanni De Maria, Maurizio Elia, Edoardo Ferlazzo, Silvana Franceschetti, Sara Gasparini, Loretta Giuliano, Angelo Labate, Angela La Neve, Adriana Magaudda, Roberto Michelucci, Francesco Paladin, Francesco Pisani, Ferdinando Sartucci, Rosalia Silvestri, Flavio Villani, Gaetano Zaccara, on behalf of the Epilepsy Study Group of the Italian Neurological Society, for their contribution to the final validation of the present manuscript and recommendations.

\section{Compliance with ethical standards}

Conflict of interest The authors declare that they have no conflict of interest.

Ethical approval None.

Informed consent None. 


\section{References}

1. Scheffer IE, Berkovic S, Capovilla G, Connolly MB, French J, Guilhoto L, Hirsch E, Jain S, Mathern GW, Moshé SL, Nordli DR, Perucca E, Tomson T, Wiebe S, Zhang YH, Zuberi SM (2017) ILAE classification of the epilepsies: position paper of the ILAE Commission for Classification and Terminology. Epilepsia 58:512-521

2. Fisher RS, Cross JH, D'Souza C et al (2017) Instruction manual for the ILAE 2017 operational classification of seizure types. Epilepsia 58:531-542

3. Smith D, Defalla BA, Chadwick DW (1999) The misdiagnosis of epilepsy and the management of refractory epilepsy in a specialist clinic. Qjm 92:15-23

4. Seneviratne U, Reutens D, D'Souza W (2010) Stereotypy of psychogenic nonepileptic seizures: insights from video-EEG monitoring. Epilepsia 51:1159-1168

5. Cho YW, Motamedi GK, Kim KT (2019) The clinical utility of non-invasive video-electroencephalographic monitoring has been diversifying. Neurol Sci 40:2625-2631

6. Berg AT, Altalib HH, Devinsky O (2017) Psychiatric and behavioral comorbidities in epilepsy: a critical reappraisal. Epilepsia 58: $1123-1130$

7. Cascino GD (2002) Clinical indications and diagnostic yield of video-electroencephalographic monitoring in patients with seizures and spells. In: Mayo Clinic Proceedings. Elsevier, pp 1111-1120

8. Ristić AJ, Drašković M, Bukumirić Z, Sokić D (2015) Reliability of the witness descriptions of epileptic seizures and psychogenic nonepileptic attacks: a comparative analysis. Neurol Res 37:560-562

9. Poushter J, Bishop C, Chwe H (2018) Social media use continues to rise in developing countries but plateaus across developed ones. Pew Res Cent 22:2-19

10. Tatum WO, Hirsch LJ, Gelfand MA, Acton EK, LaFrance WC Jr, Duckrow RB, Chen DK, Blum AS, Hixson JD, Drazkowski JF, Benbadis SR, Cascino GD, for the OSmartViE Investigators (2020) Assessment of the predictive value of outpatient smartphone videos for diagnosis of epileptic seizures. JAMA Neurol 77:593600. https://doi.org/10.1001/jamaneurol.2019.4785

11. Dash D, Sharma A, Yuvraj K, Renjith A, Mehta S, Vasantha PM, Arora A, Tripathi M (2016) Can home video facilitate diagnosis of epilepsy type in a developing country? Epilepsy Res 125:19-23. https://doi.org/10.1016/j.eplepsyres.2016.04.004

12. Huang LL, Wang YY, Liu LY, Tang HP, Zhang MN, Ma SF, Zou LP (2019) Home videos as a cost-effective tool for the diagnosis of paroxysmal events in infants: prospective study from China. J Med Internet Res 21. https://doi.org/10.2196/11229

13. Ojeda J, Gutierrez G (2016) Utility of home-made videos in an adult epilepsy clinic. J Neurol Disord 4. https://doi.org/10.4172/ 2329-6895.1000311

14. Goldenholz DM, Moss R, Jost DA, Crone NE, Krauss G, Picard R, Caborni C, Cavazos JE, Hixson J, Loddenkemper T, Salazar TD, Lubbers L, Harte-Hargrove LC, Whittemore V, Duun-Henriksen J, Dolan E, Kasturia N, Oberemk M, Cook MJ, Lehmkuhle M, Sperling MR, Shafer PO (2018) Common data elements for epilepsy mobile health systems. Epilepsia 59:1020-1026

15. Casassa C, Levit ER, Goldenholz DM (2018) Opinion and special articles: self-management in epilepsy: web-based seizure tracking applications. Neurology 91:e2027-e2030

16. Moher D, Shamseer L, Clarke $M$ et al (2015) Preferred reporting items for systematic review and meta-analysis protocols (PRISMAP) 2015 statement. Syst Rev 4:1

17. Sheth RD, Bodensteiner JB (1994) Effective utilization of homevideo recordings for the evaluation of paroxysmal events in pediatrics. Clin Pediatr (Phila) 33:578-582. https://doi.org/10.1177/ 000992289403301001
18. Samuel M, Duncan JS (1994) Use of the hand held video camcorder in the evaluation of seizures. J Neurol Neurosurg Psychiatry 57: 1417-1418. https://doi.org/10.1136/jnnp.57.11.1417

19. Johansen JR, Lindahl G, Sandstedt P (1999) Home-video observation of seizures in children with epilepsy - impact on quality of family life. Seizure 8:356-357. https://doi.org/10.1053/seiz.1999. 0305

20. Badhwar A, Siren A, Andermann E, Andermann F (2002) Myoclonic status epilepticus: video presentation. Mov Disord Off J Mov Disord Soc 17:409-411

21. Stephenson JBP, Breningstall G, Steer C, Kirkpatrick M, Horrocks I, Nechay A, Zuberi S (2004) Anoxic-epileptic seizures: home video recordings of epileptic seizures induced by syncopes. Epileptic Disord 6:15-19

22. Chen DK, Graber KD, Anderson CT, Fisher RS (2008) Sensitivity and specificity of video alone versus electroencephalography alone for the diagnosis of partial seizures. Epilepsy Behav 13:115-118. https://doi.org/10.1016/j.yebeh.2008.02.018

23. Beniczky SA, Fogarasi A, Neufeld M, Andersen NB, Wolf P, van Emde Boas W, Beniczky S (2012) Seizure semiology inferred from clinical descriptions and from video recordings. How accurate are they? Epilepsy Behav 24:213-215. https://doi.org/10.1016/j.yebeh. 2012.03.036

24. Rocha J, Pereira J (2013) Importance of video technologies in seizure identification - evidence through a case report. Int J Case Reports Med 2013:1-4. https://doi.org/10.5171/2013.656341

25. Goodwin E, Kandler RH, Alix JJP (2014) The value of home video with ambulatory EEG: a prospective service review. Seizure 23: 480-482

26. Erba $\mathrm{G}$, Giussani $\mathrm{G}$, Juersivich $\mathrm{A}$, Magaudda $\mathrm{A}$, Chiesa $\mathrm{V}$, Laganà A, di Rosa G, Bianchi E, Langfitt J, Beghi E (2016) The semiology of psychogenic nonepileptic seizures revisited: can video alone predict the diagnosis? Preliminary data from a prospective feasibility study. Epilepsia 57:777-785. https://doi.org/10.1111/epi.13351

27. Wasserman D, Herskovitz M (2017) Epileptic vs psychogenic nonepileptic seizures: a video-based survey. Epilepsy Behav 73: 42-45. https://doi.org/10.1016/j.yebeh.2017.04.020

28. Ramanujam B, Dash D, Tripathi M (2018) Can home videos made on smartphones complement video-EEG in diagnosing psychogenic nonepileptic seizures? Seizure 62:95-98. https://doi.org/10.1016/ j.seizure.2018.10.003

29. Yeh K-K, Liu W-Y, Wong AM-K, Lein R (2020) Validity of general movement assessment based on clinical and home videos. Pediatr Phys Ther 32:35-43

30. Fisher RS, Cross JH, French JA, Higurashi N, Hirsch E, Jansen FE, Lagae L, Moshé SL, Peltola J, Roulet Perez E, Scheffer IE, Zuberi SM (2017) Operational classification of seizure types by the international league against epilepsy: position paper of the ILAE Commission for Classification and Terminology. Epilepsia 58: $522-530$

31. MacDonald T, Hill A, Phan T, Fitzgerald P, Seneviratne U (2012) Psychiatry versus general physicians: who is better at differentiating epileptic from psychogenic non-epileptic seizures? Australas Psychiatry 20:379-383

32. Gedzelman ER, LaRoche SM (2014) Long-term video EEG monitoring for diagnosis of psychogenic nonepileptic seizures. Neuropsychiatr Dis Treat 10:1979

33. LaFrance WC Jr, Baker GA, Duncan R et al (2013) Minimum requirements for the diagnosis of psychogenic nonepileptic seizures: a staged approach: a report from the International League Against Epilepsy Nonepileptic Seizures Task Force. Epilepsia 54: 2005-2018

34. Engel J Jr, Wiebe S, Radhakrishnan K, Palmini A (2013) Surgical treatment for epilepsy. Neurol fachmagazin fur Neurol 2013:12-14 
35. Obeid M, Mikati MA (2007) Expanding spectrum of paroxysmal events in children: potential mimickers of epilepsy. Pediatr Neurol 37:309-316

36. Thijs RD, Bloem BR, van Dijk JG (2009) Falls, faints, fits and funny turns. J Neurol 256:155-167

37. Wirrell EC (2006) Epilepsy-related injuries. Epilepsia 47:79-86

38. Seyal M, Bateman LM (2009) Ictal apnea linked to contralateral spread of temporal lobe seizures: intracranial EEG recordings in refractory temporal lobe epilepsy. Epilepsia 50:2557-2562

39. Trinka E, Cock H, Hesdorffer D, Rossetti AO, Scheffer IE, Shinnar S, Shorvon S, Lowenstein DH (2015) A definition and classification of status epilepticus-report of the ILAE Task Force on Classification of Status Epilepticus. Epilepsia 56:1515-1523

40. Assenza G, Lanzone J, Brigo F et al (2020) Epilepsy care at the time of Covid-19 pandemic in Italy: risk factors for seizure worsening. Front Neurol 11:737

Publisher's note Springer Nature remains neutral with regard to jurisdictional claims in published maps and institutional affiliations. 